\title{
Surface roughness assessing based on digital image features
}

\author{
Simunovic, G. ${ }^{a}{ }^{,}$, Svalina, I. ${ }^{a}$, Simunovic, K. ${ }^{a}$, Saric, T. ${ }^{a}$, Havrlisan, S. ${ }^{a}$, Vukelic, D. ${ }^{b}$ \\ ${ }^{a}$ Mechanical Engineering Faculty in Slavonski Brod, University of Osijek, Croatia \\ ${ }^{b}$ Faculty of Technical Sciences, University of Novi Sad, Serbia
}

\begin{abstract}
A B S T R A C T
The paper gives an account of the machined surface roughness investigation based on the features of a digital image taken subsequent to the technological operation of milling of aluminium alloy Al6060. The data used for investigation were obtained by mixed-level factorial design with two replicates. Input variables (factors) are represented by the face milling basic machining parameters: spindle speed (at five levels: 2000; 3500; 5000; 6500; 8000 $\mathrm{rev} / \mathrm{min}$, respectively), feed per tooth (at six levels: $0.025 ; 0.1 ; 0.175 ; 0.25$; $0.325 ; 0.4 \mathrm{~mm} /$ tooth, respectively) and depth of cut (at two levels: $1 ; 2 \mathrm{~mm}$, respectively). Output variable or response is the most frequently used surface roughness parameter - arithmetic average of the roughness profile, Ra. Digital image of the machined surface is provided for every test sample. Based on experimental design and obtained results of roughness measuring, a base has been created of input data (features) extracted from digital images of the samples' machined surfaces. This base was later used for generating the fuzzy inference system for prediction of the surface roughness using the adaptive neuro-fuzzy inference system (ANFIS). Assessing error, i.e. comparison of the assessed value $R a$ provided by the system with real $R a$ values, is expressed with the normalized root mean square error (NRMSE) and it is 0.0698 (6.98\%).
\end{abstract}

(C) 2016 PEI, University of Maribor. All rights reserved.

\author{
ARTICLE INFO \\ Keywords: \\ Surface roughness \\ Face milling \\ Digital image \\ Adaptive neuro-fuzzy inference \\ system
}

*Corresponding author:

goran.simunovic@sfsb.hr

(Simunovic, G.)

Article history:

Received 7 March 2016

Revised 15 May 2016

Accepted 16 May 2016

\section{References}

[1] Stankovic, I., Perinic, M., Jurkovic, Z., Mandic, V., Maricic, S. (2012). Usage of neural network for the prediction of surface roughness after the roller burnishing, Metalurgija, Vol. 51, No. 2, 207-210.

[2] Simunovic, K., Simunovic, G., Saric, T. (2015). Single and multiple goal optimization of structural steel face milling process considering different methods of cooling/lubricating, Journal of Cleaner Production, Vol. 94, 321-329, doi: 10.1016/i.jclepro.2015.02.015.

[3] Zuperl, U., Cus, F. (2015). Simulation and visual control of chip size for constant surface roughness, International Journal of Simulation Modelling, Vol. 14, No. 3, 392-403, doi: 10.2507/IJSIMM14(3)2.282.

[4] Vukelic, D., Tadic, B., Miljanic, D., Budak, I., Todorovic, P.M., Randjelovic, S., Jeremic, B.M. (2012). Novel workpiece clamping method for increased machining performance, Tehnički vjesnik - Technical Gazette, Vol. 19, No. 4, 837846.

[5] Brezocnik, M., Kovacic, M., Ficko, M. (2004). Prediction of surface roughness with genetic programming, Journal of Materials Processing Technology, Vol. 157-158, 28-36, doi: 10.1016/j.jmatprotec.2004.09.004.

[6] Pare, V., Agnihotri, G., Krishna, C. (2015). Selection of optimum process parameters in high speed CNC endmilling of composite materials using meta heuristic techniques - A comparative study, Strojniški vestnik - Journal of Mechanical Engineering, Vol. 61, No. 2, 176-186, doi: 10.5545/sv-jme.2014.1914.

[7] Simunovic, G., Simunovic, K., Saric, T. (2013). Modelling and simulation of surface roughness in face milling, International Journal of Simulation Modelling, Vol. 12, No. 3, 141-153, doi: 10.2507/IJSIMM12(3)1.219.

[8] Çolak, O. (2014). Optimization of machining performance in high-pressure assisted turning of Ti6Al4V alloy, Strojniški vestnik - Journal of Mechanical Engineering, Vol. 60, No. 10, 675-681, doi: 10.5545/sv-jme.2014.1914. 
[9] Nammi, S., Ramamoorthy, B. (2014). Effect of surface lay in the surface roughness evaluation using machine vision, Optik - International Journal for Light and Electron Optics, Vol. 125, No. 15, 3954-3960, doi: 10.1016/ j.ijleo.2014.01.152.

[10] Klancnik, S., Ficko, M., Balic J., Pahole, I. (2015). Computer vision-based approach to end mill tool monitoring, International Journal of Simulation Modelling, Vol. 14, No. 4, 571-583, doi: 10.2507/IJSIMM14(4)1.301.

[11] Krolczyk, G., Raos, P., Legutko, S. (2014). Experimental analysis of surface roughness and surface texture of machined and fused deposition modelled parts, Tehnički vjesnik - Technical Gazette, Vol. 21, No. 1, 217-221.

[12] Samtaş, G. (2014). Measurement and evaluation of surface roughness based on optic system using image processing and artificial neural network, The International Journal of Advanced Manufacturing Technology, Vol. 73, No. 1, 353-364, doi: 10.1007/s00170-014-5828-1.

[13] Stępień, K., Makiela, W., Stoić, A., Samardžić, I. (2015). Defining the criteria to select the wavelet type for the assessment of surface quality, Tehnički vjesnik - Technical Gazette, Vol. 22, No. 3, 781-784, doi: 10.17559/TV20140124110406.

[14] Lee, K.C., Ho, S.J., Ho, S.Y. (2005). Accurate estimation of surface roughness from texture features of the surface image using an adaptive neuro-fuzzy inference system, Precision Engineering, Vol. 29, No. 1, 95-100, doi: 10.1016/j.precisioneng.2004.05.002.

[15] Jeyapoovan, T., Murugan, M. (2013). Surface roughness classification using image processing, Measurement, Vol. 46, No. 7, 2065-2072, doi: 10.1016/i.measurement.2013.03.014.

[16] Morala-Argüello, P., Barreiro, J., Alegre, E. (2012). A evaluation of surface roughness classes by computer vision using wavelet transform in the frequency domain, The International Journal of Advanced Manufacturing Technology, Vol. 59, No. 1, 213-220, doi: 10.1007/s00170-011-3480-6.

[17] Palani, S., Natarajan, U. (2011). Prediction of surface roughness in CNC end milling by machine vision system using artificial neural network based on 2D Fourier transform, The International Journal of Advanced Manufacturing Technology, Vol. 54, No. 9, 1033-1042, doi: 10.1007/s00170-010-3018-3.

[18] Ho, S.Y., Lee, K.C., Chen, S.S., Ho, S.J. (2002). Accurate modeling and prediction of surface roughness by computer vision in turning operations using an adaptive neuro-fuzzy inference system, International Journal of Machine Tools and Manufacture, Vol. 42, No. 13, 1441-1446, doi: 10.1016/S0890-6955(02)00078-0.

[19] Palani, S., Natarajan, U., Chellamalai, M. (2013). On-line prediction of micro-turning multi-response variables by machine vision system using adaptive neuro-fuzzy inference system (ANFIS), Machine Vision and Applications, Vol. 24, No. 1, 19-32, doi: 10.1007/s00138-011-0378-0.

[20] Natarajan, U., Palani, S., Anandampilai, B. (2012). Prediction of surface roughness in milling by machine vision using ANFIS, Computer-Aided Design \& Applications, Vol. 9, No. 3, 269-288, doi: 10.3722/cadaps.2012.269-288.

[21] Jeyapoovan, T., Murugan, M. (2013). Surface roughness classification using image processing, Measurement, Vol. 46, No. 7, 2065-2072, doi: 10.1016/i.measurement.2013.03.014.

[22] Zawada-Tomkiewicz, A. (2010). Estimation of surface roughness parameter based on machined surface image, Metrology and Measurement Systems, Vol. 17, No. 3, 493-504.

[23] Priya, P., Ramamoorthy, B. (2007). The influence of component inclination on surface finish evaluation using digital image processing, International Journal of Machine Tools and Manufacture, Vol. 47, No. 3-4, 570-579, doi: 10.1016/i.ijmachtools.2006.05.005.

[24] Lee, B.Y., Yu, S.F., Juan, H. (2004). The model of surface roughness inspection by vision system in turning, Mechatronics, Vol. 14, No. 1, 129-141, doi: 10.1016/S0957-4158(02)00096-X.

[25] Gadelmawla, E.S., Al-Mufadi, F.A., Al-Aboodi, A.S. (2014). Calculation of the machining time of cutting tools from captured images of machined parts using image texture features, Proceedings of the Institution of Mechanical Engineers, Part B: Journal of Engineering Manufacture, Vol. 228, No. 2, 203-214, doi: 10.1177/0954405413481291.

[26] Dutta, S., Datta, A., Das Chakladar, N., Pal, S.K., Mukhopadhyay, S., Sen, R. (2012). Detection of tool condition from the turned surface images using an accurate grey level co-occurrence technique, Precision Engineering, Vol. 36, No. 3, 458-466, doi: 10.1016/j.precisioneng.2012.02.004.

[27] Shahabi, H.H., Ratnam, M.M. (2009). In-cycle monitoring of tool nose wear and surface roughness of turned parts using machine vision, The International Journal of Advanced Manufacturing Technology, Vol. 40, No. 11, 11481157, doi: 10.1007/s00170-008-1430-8.

[28] Nathan, D., Thanigaiyarasu, G., Vani, K. (2014). Study on the relationship between surface roughness of AA6061 alloy end milling and image texture features of milled surface, Procedia Engineering, Vol. 97, 150-157, doi: 10.1016/i.proeng.2014.12.236.

[29] Kamguem, R., Tahan, S.A., Songmene, V. (2013). Evaluation of machined part surface roughness using image texture gradient factor, International Journal of Precision Engineering and Manufacturing, Vol. 14, No. 2, 183-190, doi: $10.1007 / \mathrm{s} 12541-013-0026-\mathrm{x}$. 
APEM
Advances in Production Engineering \& Management

Letnik 11 | Številka 2 | Junij 2016 | pp 93-104

http://dx.doi.org/10.14743/apem2016.2.212
ISSN 1854-6250

Spletna stran: apem-journal.org

Izvirni znanstveni članek

\title{
Ocenitev površinske hrapavosti na osnovi lastnosti digitalnih slik
}

\author{
Simunovic, G. ${ }^{a}{ }^{,}{ }^{,}$, Svalina, I. ${ }^{a}$, Simunovic, K. ${ }^{a}$, Saric, T. ${ }^{a}$, Havrlisan, S. ${ }^{a}$, Vukelic, D. ${ }^{b}$ \\ ${ }^{a}$ Mechanical Engineering Faculty in Slavonski Brod, University of Osijek, Croatia \\ ${ }^{\mathrm{b}}$ Faculty of Technical Sciences, University of Novi Sad, Serbia
}

\section{POVZETEK}

$\mathrm{V}$ prispevku je podana raziskava površinske hrapavosti na osnovi digitalnih slik, ki so bile zajete po tehnološki operaciji rezkanja preizkušanca iz zlitine Al6060. Podatki, ki so bili uporabljeni pri raziskavi, so bili dobljeni s faktorskim načrtom, in sicer z različnimi vrednostmi (nivoji) in z dvema ponovitvama. Vhodne spremenljivke (faktorji) so bili osnovni parametri pri čelnem rezkanju: hitrost vrtenja vretena (pet nivojev: 2000, 3500, 5000, 6500 in 8000 rpm), podajanje na zob (šest nivojev: $0.025,0.1,0.175,0.25,0.325,0.4$ $\mathrm{mm} / \mathrm{zob}$ ) in globina reza (dva nivoja: 1 in $2 \mathrm{~mm}$ ). Izhodna spremenljivka (odziv) je bila aritmetično povprečje profila hrapavosti $R a$. Za vsak vzorec je bila dobljena digitalna slika obdelane površine. Na osnovi načrtovanja eksperimentov in dobljenih rezultatov je bila narejena baza vhodnih podatkov (značilnosti) pridobljenih iz digitalnih slik obdelanih površin. Ta baza je bila nato uporabljena za obdelavo $\mathrm{z}$ adaptivnim nevro-mehkim inferenčnim sistemov (ANFIS). Ocenjena napaka, tj. primerjava med vrednostjo $R a$, pridobljeno s pomočjo sistema ANFIS z resnično vrednostjo $R a$, je bila izražena $\mathrm{z}$ normalizirano srednjo kvadratno napako (NRMSE), ki je znašala 0.0698 oziroma $6.98 \%$.

\section{PODATKI O ČLANKU}

Ključne besede: Površinska hrapavost

Čelno rezkanje

Digitalna slika

Adaptivni nevro-mehki inferenčni sistem

*Kontaktna oseba: goran.simunovic@sfsb.hr (Simunovic, G.)

Zgodovina članka:

Prejet 7. marca 2016

Popravljen 15. maja 2016

Sprejet 16. maja 2016 